\title{
Feature selection for gesture recognition in Internet-of-Things for healthcare
}

\author{
Giulia Cisotto $^{1,2}$, Martina Capuzzo ${ }^{1}$, Anna V. Guglielmi ${ }^{1}$, Andrea Zanella ${ }^{1}$ \\ ${ }^{1}$ Dept. of Information Engineering, University of Padova, Padova, Italy \\ ${ }^{2}$ Integrative Brain Imaging Center, National Center of Neurology and Psychiatry, Tokyo, Japan \\ email: \{cisottog, capuzzom, guglielm, zanella\}@ dei.unipd.it
}

\begin{abstract}
Internet of Things is rapidly spreading across several fields, including healthcare, posing relevant questions related to communication capabilities, energy efficiency and sensors unobtrusiveness. Particularly, in the context of recognition of gestures, e.g., grasping of different objects, brain and muscular activity could be simultaneously recorded via EEG and EMG, respectively, and analyzed to identify the gesture that is being accomplished, and the quality of its performance. This paper proposes a new algorithm that aims (i) to robustly extract the most relevant features to classify different grasping tasks, and (ii) to retain the natural meaning of the selected features. This, in turn, gives the opportunity to simplify the recording setup to minimize the data traffic over the communication network, including Internet, and provide physiologically significant features for medical interpretation. The algorithm robustness is ensured both by consensus clustering as a feature selection strategy, and by nested cross-validation scheme to evaluate its classification performance. Although Feature Selection with Consensus (FeSC) implements a very robust architecture for feature selection and classification, results are still negatively affected by the limited size of the dataset. In the future, further investigations could determine to what extent size could cause a drop in the performance of $\mathrm{FeSC}$ in this and other gesture recognition applications.
\end{abstract}

Index Terms-Feature selection, EEG, EMG, consensus clustering, nested cross-validation, Internet-of-Things for Healthcare.

\section{INTRODUCTION}

I NTERNET-of-Things is pervasively entering every field of society, including healthcare. Indeed, in the so-called Internet-of-Things for healthcare (IoTH) [1], several heterogeneous sensors, portable devices and wearables could be employed to continuously acquire large amounts of physiological data (e.g., heart rate, blood pressure and brain activity) as well as living context and environmental variables (e.g., artificial light exposure, air quality and (acoustic) noise level). Among other healthcare applications, gesture recognition can be evaluated by simultaneously recording brain and muscular activities via electroencephalography (EEG) and electromyography (EMG), respectively, and analyzing them to identify the specific movement that is being accomplished and the quality of its performance. It could be useful for assessing the advancements of training in patients recovering from neuro-motor diseases (e.g., stroke) or in athletes, where technical and complex gestures have to be optimally performed for a competition or recovered after an injury [2]. However, wearing tens of sensors on the top of the head (to acquire brain activity) and along the limbs (to acquire muscular activity) can not be comfortable nor useful: unobtrusiveness and minimal setups are highly recommended in order not to interfere with the natural gestures and to ensure subject compliance. Moreover, an increasing number of IoT applications is being developed with the result that a growing amount of data is circulating the network, possibly leading to saturate the channel capacity and dramatically decrease the quality of service (QoS) of network users. Again, minimizing the acquisition setup and thus, the amount of data to acquire and process could represent a key solution to enabling a sustainable development of an IoT for healthcare (besides increasing the communication networks performance using new generations of communications technologies). Therefore, in this paper we propose a robust feature selection algorithm, namely Feature Selection with Consensus (FeSC) for EEG/EMG-based gesture recognition (i) to robustly extract the most relevant features to classifying different grasping tasks, minimizing the acquisition setup to those EEG/EMG sensors that only convey significant information and (ii) to retain the natural meaning of the selected features.

The paper is organized as follows. In Sec. II we present the related works; in Sec. III. we describe how individual features are computed from EEG and EMG signals. Sec. IV explains the details of the proposed feature selection algorithm. Sec. V reports the results obtained during preliminary investigations on a publicly available dataset. The novelty of FeSC and its most significant differences and advantages compared to the state-of-art are discussed in Sec. VI. Finally, Sec. VII concludes the paper, also mentioning possible extensions to this work.

\section{RELATED WORKS}

Feature selection is becoming critical in several applications of IoTH, e.g., gesture recognition, emotion recognition and sleep/wake detection among others [3][5]. Particularly, finding a small number of highly discriminative features to compress the available big data could bring significant improvements in the classification performance [6], [7] and the reduction of the required communication resources. Feature selection has been explored in many studies $[8]-[11]$, where several implementations of sequential forward/backward selection/elimination algorithms have been presented. Among other methods, hierarchical clustering was found to be a common choice to progressively reduce the number of features. In [8], authors study the relevance of different feature combinations, thus providing an improvement of the accuracy performance using several classifiers. [9] uses mutual information and the coefficient of relevancy to measure the distance between and within clusters, respectively. In [10], 
authors survey different methods for feature selection and describe several evaluation measures that can be used to compare their performance. Interestingly, they point out that clustering-based feature selection methods could retain irrelevant features: indeed, the latter are typically clustered together, forming large clusters which might be then represented by a representative feature(s) in the final set of selected features. As a solution, [11] applies consensus clustering algorithm where multiple runs with different initializations of the clustering algorithm are combined together to return a smaller, but more robust, set of features. In [11], consensus clustering has been applied to neuroimaging signals, with the aim to reduce the spatial complexity of that dataset, while retaining the anatomical meaning of the signals. In our work we apply a similar approach but, as input to the consensus algorithm, we take the outputs of two different clustering algorithms (i.e., hierarchical and spectral clustering) and, as main novelty, we introduce consensus clustering to cluster EEG,EMG features (themselves) together. Furthermore, we take advantage of the well-established physiological value of the magnitude squared coherence (MSC), a feature extracted from the joint analysis of EEG and EMG signals. While the most literature on feature selection from heterogeneous signals (e.g., EEG, EMG, electroculography (EOG), electrocardiography (ECG) and others) deals with unimodal features, i.e., extracted from one single kind of signal at a time, or artificial features, i.e., extracted using deep learning architectures [12], we consider a well-known multimodal feature that allows us to preliminary, and significantly, reduce the overall number of available features, while retaining, at the same time, their physiological relevance [7]. Moreover, other previous works often limit the number of features by manually selecting, a-priori, regions of interest (ROI) (i.e., sensors) where features are extracted from. Here, instead, we use an extensive approach where all EEG and EMG sensors are taken into account and selected manually.

\section{DATA PRE-PROCESSING}

In this section we describe the feature extraction and the dataset augmentation pre-processing steps. Given a pair of single-channel EEG and EMG signals, i.e., acquired from the $h$-th (out of $H$ ) EEG sensor and from the $j$ th (out of $J$ ) EMG sensor, respectively, let $s_{x}^{i}(t)$ and $s_{y}^{i}(t)$, with $i=1,2, \ldots . N$, represent all EEG and EMG segments, respectively, corresponding to $N$ repetitions (trials) of the gesture to investigate. Let $S_{x}^{i}(f)$ and $S_{y}^{i}(f)$, with $i=1,2, \ldots N$, represent the autospectra of the $i$-th segment of EEG and EMG, respectively, at repetition $i$. Also, $S_{x y}^{i}(f)$ is the EEG-EMG (cross) power spectrum of the $i$-th segment. The Fast Fourier Transform (FFT) algorithm is used to compute the spectra of segments. Then, $S_{x}(f), S_{y}(f)$, and $S_{x y}(f)$ are the averages along the $N$ repetitions, obtained from $S_{x}^{i}(f), S_{y}^{i}(f)$, and $S_{x y}^{i}(f)$, respectively, with $i=1,2, \ldots, N$. Finally, $\mathbf{M S C}_{i j}[13]$ is the normalized $h$-th EEG- $j$-th EMG (cross) power spectrum, after averaging along repetitions, and it is obtained as follows:

$$
M S C_{h j}(f)=\frac{\left|S_{x y}(f)\right|^{2}}{S_{x}(f) S_{y}(f)}
$$

Given the Cauchy-Schwarz inequality, it holds

$$
0 \leq\left|S_{x y}(f)\right|^{2} \leq S_{x}(f) S_{y}(f)
$$

and, as a consequence, $M S C_{h j}(f)$ assumes values between 0 (uncorrelated signals) and 1 (perfect linear relationship). From the MSC spectrum of each pair of EEGEMG signals, we then extract the average MSC value of $K=11$ well-known different frequency bands: $\delta$ $(1.5-4 \mathrm{~Hz}), \theta(4-8 \mathrm{~Hz}), \alpha(8-13 \mathrm{~Hz}), \beta_{1}(13-20 \mathrm{~Hz})$, $\beta_{2}(20-30 \mathrm{~Hz}), \beta(13-30 \mathrm{~Hz}), \gamma_{1}(30-45 \mathrm{~Hz}), \gamma_{2}(45-$ $60 \mathrm{~Hz}), \gamma_{3}(60-80 \mathrm{~Hz}), \gamma(30-80 \mathrm{~Hz})$, and the full band $(1.5-80 \mathrm{~Hz})$. It is worth noting that some of them are overlapping with each other, e.g., $\beta_{1}$ and $\beta$, while others cover multiple narrower sub-bands, e.g., $\beta$ includes $\beta_{1}$ and $\beta_{2}$. All possible EEG-EMG-frequency band triplets, i.e., $\mathcal{M}=\left\{E E G_{h}, E M G_{j}\right.$, band $\left._{k}\right\}$, with $h=1,2, \ldots . H$, $j=1,2, \ldots . J$, and $k=1,2, \ldots . K$ have been included into the feature matrix. The same feature extraction procedure is repeated for all repetitions of all types (i.e., classes) of motor task. Finally, to obtain balanced classes for a fair classification between different classes, we apply SMOTE to the smallest class [14]. Provided that a highdimensional feature matrix is typically extracted at this step, an automatic procedure is needed to select the most relevant features for obtaining a reduced dataset (data mining) and to improving the classification performance.

\section{Proposed feAture SELECTION ALGORIthM}

The aim of FeSC is two-fold: first, to reduce the number of features, keeping only the most informative ones using consensus clustering and, second, to select those which give the best performance in the classification of different grasping tasks. Nested cross-validation (CV) is implemented to realize a robust validation strategy [15]. To ensure a proper performance evaluation, $10 \%$ of the dataset is kept for the final independent test, while the remaining $90 \%$ is used for the nested procedure. Every partition used in FeSC includes the same proportion of class instances as the overall dataset. FeSC pipeline is represented in Fig. 1] block $A$ represents the consensus clustering, block $B$ is for data mining and classification, blocks $C 1$ and $C 2$ include the inner and outer $\mathrm{CV}$ loops, respectively, and block $D$ performs grid-search parameter optimization. In the following, we illustrate how the pipeline works, referring to Figs. 1, 2 ,

\section{A. Consensus clustering}

The algorithm for consensus clustering is represented by block $A$ in Fig. 1 and is further expanded in Fig. 2, which can be used as a reference for the following description. We employ a consensus-based feature selection approach similar to what presented in [11]. The input is defined as a vector $\gamma_{r}=\left[M_{r}, \sigma_{r}, \nu_{r}\right]$, with $r=1,2, \ldots R$ formed by the following clustering parameters: $M_{r}$, the maximum number of clusters on which the consensus algorithm works, i.e., that also corresponds to the number of features we decide to extract, and the thresholds $\sigma_{r}$ and $\nu_{r}$. The main steps for the consensus clustering are the following:

1) Application of two clustering algorithms (agglomerative hierarchical and spectral clustering), separately, to the initial dataset; 


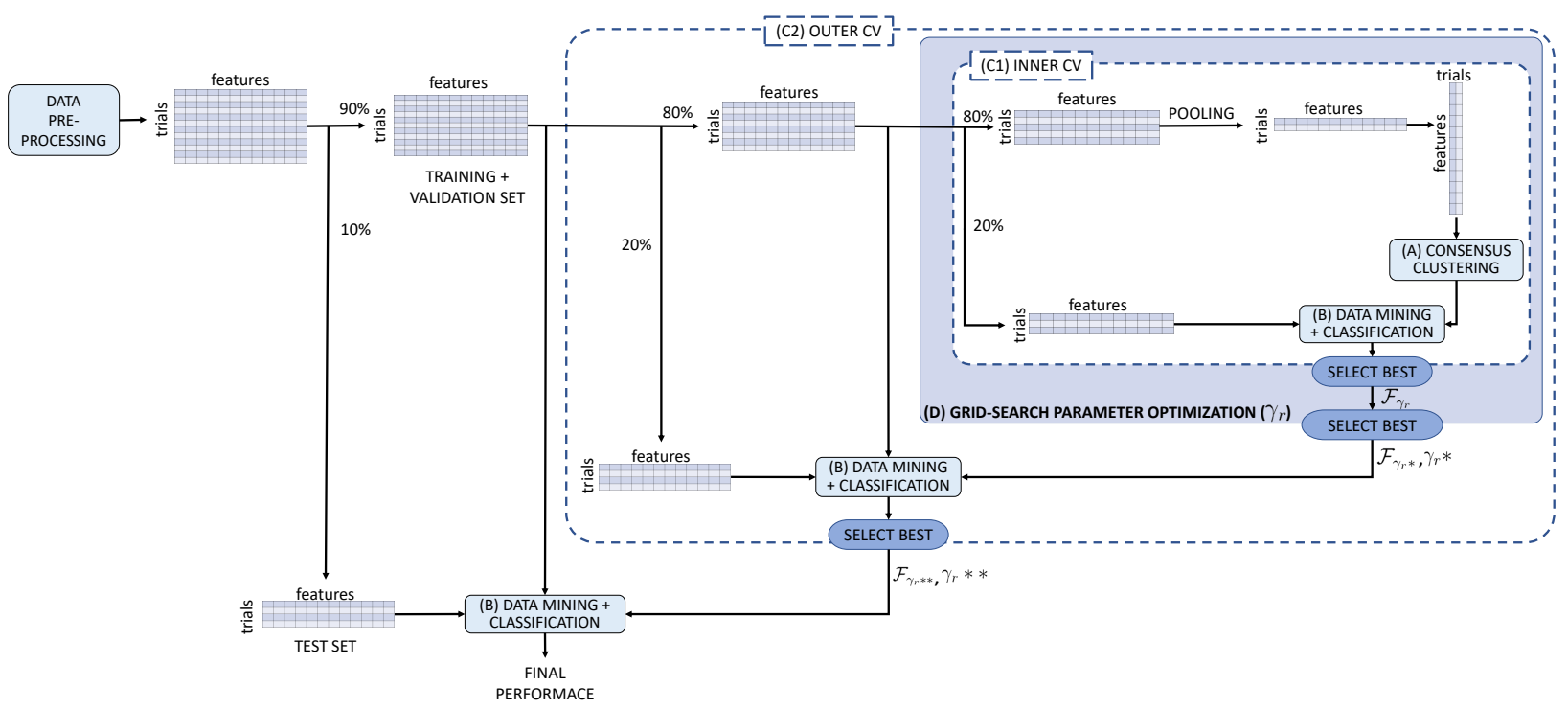

Fig. 1: FeSC: the proposed feature selection algorithm.

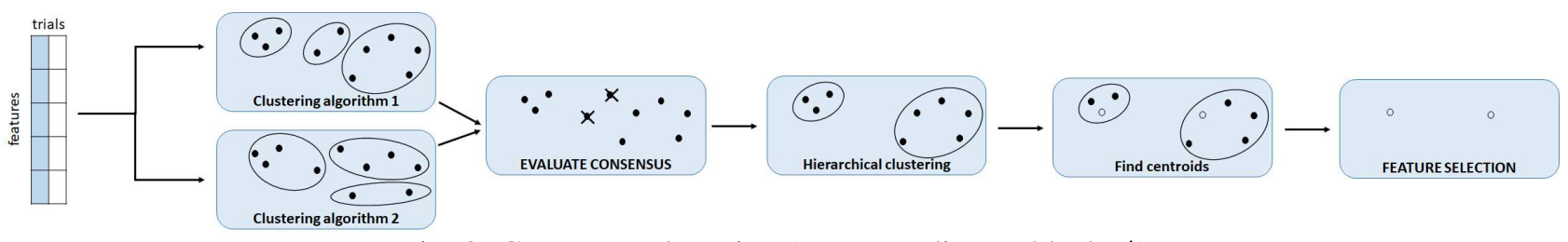

Fig. 2: Consensus clustering (corresponding to block $A$ ).

2) Evaluation of the consensus between them. This step is further divided into three substeps:

i. Computation of a pair-wise similarity matrix for each clustering algorithm, indicating if two features are assigned to the same cluster.

ii. Computation of a consensus matrix, which takes the average of the similarity matrices. In this matrix, the higher the value associated to a pair of features, the more often these features are clustered together by the different clustering algorithms.

iii. Consensus-based filtering: application of thresholds $\left(\sigma_{r}\right.$ and $\left.\nu_{r}\right)$ to the consensus matrix in order to select a subset of features (i.e., candidate to be finally selected). Given that $\sigma_{r}$ is related to the agreement between the different clustering algorithms, and $\nu_{r}$ is related to the average size of clusters, the features which have similarity greater than $\sigma_{r}$ with more than $\nu_{r}$ other features are only considered.

3) Hierarchical clustering of features identified at the previous step, setting the number of clusters to $M_{r}$.

4) Computation of the centroids of clusters found at the previous step: these are taken as the selected features.

As mentioned before, we consider two algorithms for the consensus (step 1): agglomerative hierarchical clustering and spectral clustering.

\section{Agglomerative hierachical clustering}

Hierarchical clustering aims at grouping data over a variety of scales by creating a cluster tree called dendrogram [16]. The dendrogram is a multilevel hierarchy in which clusters at one level are joined as clusters of the next level. In this way, it is possible to decide the most appropriate level of clustering. The hierarchical clustering algorithm works in the following way. First, it computes the distance between objects in the dataset based on a certain metric. These distances are sorted in ascending order and objects with minimum distance are clustered together. Once objects are paired in binary clusters, these are paired in larger clusters. This procedure is repeated until all objects are linked together in a hierarchical tree. Finally, the hierarchical tree is cut in order to define the output clusters: to create a partition of the initial dataset, the branches at the bottom of the hierarchical tree are pruned off and all objects below each cut are assigned to a single cluster. In our application, we use Euclidean distance and Ward's method to group clusters; as cutting rule, we set the number of clusters to be equal to $M_{r}$.

\section{Spectral clustering}

Spectral clustering was first introduced in [17], where authors proposed to make a partition in a graph based on eigenvectors (i.e., the "spectrum") of the similarity matrix of the data. In general, spectral clustering refers to a class of techniques relying on the eigenstructure of a similarity matrix to partition points into disjoint clusters: points having high similarity are assigned to the same cluster. For our scope, we consider the spectral clustering algorithm presented in [18], which employs the adjacency matrix, i.e., a matrix representing a graph, where elements indicate whether pairs of vertices are adjacent in the graph or not. The algorithm consists of two main steps:

1) Transformation of the adjacency matrix of the input data into a matrix with as many rows as the input 
elements and $M_{r}$ columns corresponding to the first $M_{r}$ eigenvectors.

2) Application of the $k$-means algorithm [19] to clusterize the rows of the matrix above defined. Then, the output of the algorithm represents the $M_{r}$ clusters of the input elements.

The success of spectral clustering is mainly based on the fact that no assumptions on the shape of clusters are needed. Moreover, it can be implemented efficiently even for large datasets, as long as the similarity graph is sparse. Indeed, once the similarity graph is chosen, the main step in the matrix transformation is the computation of eigenvectors which, being a linear problem, is amenable to simple and deterministic solution. However, the choice of a good similarity graph is, in general, not trivial.

\section{B. Data mining and classification}

In these blocks we assess the classification performance over the current training set using the current selection of features. Therefore, we consider the validation set and apply data mining to retain only the features that are selected by the consensus clustering procedure. Then, we classify heavy/light trials by applying Support Vector Machine (SVM), SVM are models for supervised learning in (binary) classification and regression problems [19]. The input elements can be represented as points in the feature space and the classifier as a hyperplane that is expected to optimally separate them. During the training phase, the SVM builds a non-probabilistic classifier to distinguish the two classes. In the second phase (test phase), new elements (belonging to the current validation set) are provided to the model and mapped in the same feature space: according to which side of the hyperplane they are located, they are classified into a class or the other. In our application, we consider an $M_{r}$-dimensional space and accuracy as main performance metric. Furthermore, with the kernel trick it is possible to obtain nonlinear SVM, here, we compare the performance obtained using either a linear or a radial basis function (rbf) kernel.

\section{C1. Inner cross-validation}

For the inner CV we use a 5-fold CV (see Fig. 1 block $C 1$ ). It consists in partitioning the dataset in 5 groups (folds), training the model on 4 folds, and finally testing it in the remaining fold. The training-test procedure is run 5 times, changing the fold that is used for validation at every iteration and keeping track of the evaluation performance. Before applying the consensus clustering procedure, we perform a pooling operation to reduce in a two-dimensional space the elements that characterize each feature: specifically, we take the mean of MSC across all trials of the same class $\left(\overline{M S C}_{\text {heavy }}, M S C_{\text {light }}\right)$. Therefore, each feature is now described by a vector $\left[\overline{M S C}_{\text {heavy }}, \overline{M S C}_{\text {light }}\right]$. We apply the consensus clustering as described in Sec. IV-A and obtain one specific selection of features for each iteration of the inner $\mathrm{CV}$. Then, we evaluate the classification performance using a kernel-SVM classifier, as explained in Sec. IV-B. Finally, we select the $\mathcal{F}_{\gamma_{r}}$ set of features which provides the best classification accuracy for the current combination of parameters $\gamma_{r}$. The procedure of this block is repeated for every combination of parameters.

\section{Grid-search parameter optimization}

As sketched in Fig. 1, grid-search parameter optimization is implemented to find the optimal combination of consensus clustering parameters that leads to the best classification performance with the minimum number of features. Therefore, the inner $\overline{C V}$ is repeated for every $\gamma_{r}$. We select the $\gamma_{r} *$ that achieves the best classification performance. If the same accuracy is obtained by two different inner folds (i.e., possibly two different selection of $M_{r}$ features), we retain that with the minimum number of selected features. The outputs of this block are $\gamma_{r} *$ and the set of best features $\mathcal{F}_{\gamma_{r}}^{*}$.

\section{C2. Outer $C V$}

For the outer CV, we use a 5-fold CV (see Fig. 1, block $C 2$ ). At each iteration, four folds are input to the gridsearch parameter optimization (block $D$ ) and the inner $\mathrm{CV}$ (block $C 1$ ). The fifth fold is used as validation set to evaluate the model selected in block $D$. As previously (see Section IV-B], we select the fold associated with the highest accuracy. Therefore, the outputs of this block are $\gamma_{r}^{* *}$ and the set of best features $\mathcal{F}_{\gamma_{r}}^{* *}$.

\section{Final performance evaluation}

Then, we use the selection of features $\mathcal{F}_{\gamma_{r}^{* *}}$ for the data mining step and the SVM training (block $B$ ), using the current training set (i.e., $90 \%$ of the original dataset). Finally, we test this model to classify heavy/light trials in the independent test set ( $10 \%$ of the original dataset).

\section{Results}

\section{A. The dataset}

The dataset is extracted from the large, publiclyavailable, WAY-EEG-GAL dataset [20]. EEG and EMG data (simultaneously recorded) were obtained while partecipants were performing repetitions (i.e., trials) of a graspand-lift task. At each repetition, they had to grasp an object with their thumb and index fingers, lift it up to an a-priori selected position, hold it for a few seconds, and then return to the initial position, releasing the object. Different grasp-and-lift tasks were randomly proposed to the participants: the object could be changed in weight (i.e., light $=165 \mathrm{~g}$, medium $=330 \mathrm{~g}$, and heavy $=660$ g), surface friction (sandpaper, suede, and silk) or both. Here, we consider all repetitions where the object was light, as class 1 , and all repetitions where the object was heavy, as class 2, regardless to their surface friction. Thirty-two EEG signals were acquired from thirty-two sensors, placed at standard locations (according to the International 10-20 EEG System). Moreover, ten EMG sensors were placed over five different muscles of the right upper limb, thus acquiring five bipolar EMG signals. Data from 8 participants were included in this work (those without recodings issues). Then, we extracted 672 trials with light objects, 456 trials with heavy objects, 393 trials with sandpaper surface objects, and 1754 trials with silk surface objects. In this work, we classified light vs heavy trials. 

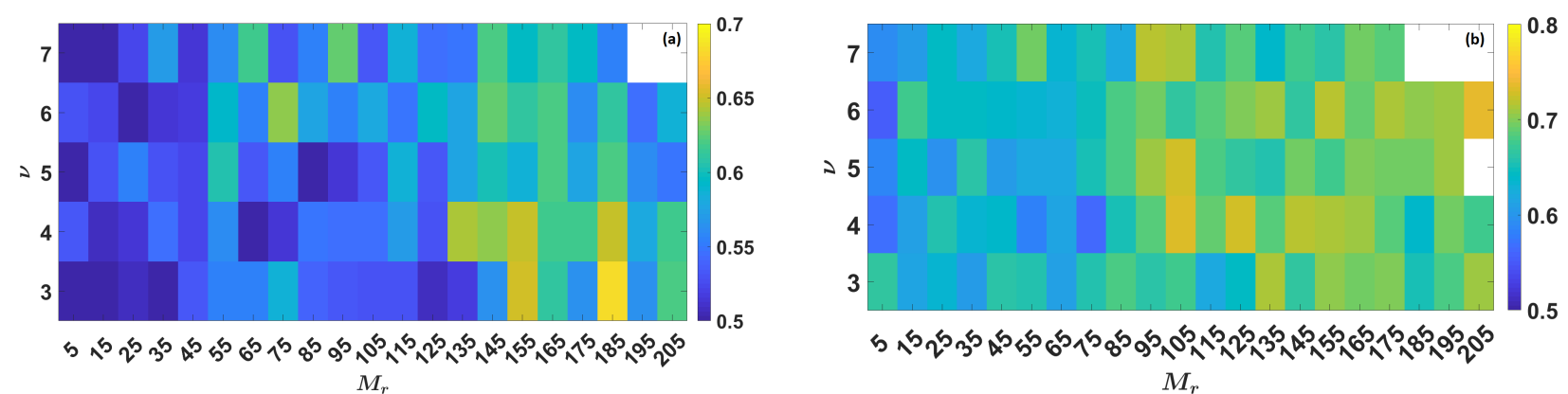

Fig. 3: Training performance in the inner $\mathrm{CV}$ fold giving $\gamma_{r}^{* *}$ and $\mathcal{F}_{\gamma_{r}^{* *}}$ with variable number of features $\left(M_{r}\right)$ and $\nu$ values. (a) Linear kernel. (b) Rbf kernel.

\begin{tabular}{cccc}
\hline Kernel & Parameters tuning & Training & Final \\
\hline Radial Basis Function & {$[135,0.6,3]$} & 0.6570 & 0.6045 \\
\hline Linear & {$[195,0.6,3]$} & 0.5909 & 0.4851 \\
\hline
\end{tabular}

TABLE I: Training and test FeSC classification performance.

\section{B. Pre-processing}

According to the experimental records available online, all data were artefacts-free. Therefore, we performed a lightweight pre-processing. Particularly, the EMG signals were downsampled to $500 \mathrm{~Hz}$ (same sampling frequency as the EEG). Then, we used bandpass filtering (Chebyshev type I, order 86) for EEG and EMG to limit the signals in the frequency band $1.5-80 \mathrm{~Hz}$. Moreover, we used notch filter (IIR filter, direct-form II, order 2) to remove the power supply component in both signals. The filtered signals were segmented into $4 \mathrm{~s}$ long segments, each one corresponding to a single repetition of the graspand-lift task. EMG segments were full-wave rectified. Then, all segments were normalized over their own areaunder-curve (AUC) value. Finally, MSC was computed, as explained in Sec. III, for each segment, resulting in a dataset of $5 \times 32 \times 11$ (1760) features with 456 trials for heavy objects and 672 trials for light objects. As suggested by [14], we scale the input feature matrix and we applied SMOTE (see Sec. III) to obtain balanced classes. Then, we obtain a normalized input feature matrix with 672 trials for both classes. FeSC pipeline has been fully implemented in Matlab and made available on GitHub 1

\section{Parameters optimization and performance evaluation}

Fig. 3 shows the training performance obtained in the inner $\mathrm{CV}$ (block $C 1$ ) for all combinations of clustering parameters $\gamma_{r}=\left[M_{r}, \sigma_{r}, \nu_{r}\right]$ with two different kernel types, i.e., linear and rbf. Note that, since only two clustering algorithms have been used in the consensus clustering step, $\sigma_{r}$ is kept fixed to 0.6 (i.e., to realize an actual consensus more than half clustering algorithms should agree on clustering two features together). Blank regions correspond to parameter combinations where consensus could not be found, while colored pixels represent reliable parameters combinations. It is worth noting that Fig. 3 only reports results from one single inner $\mathrm{CV}$ iteration, i.e., the one providing $\gamma_{r} *$ (for both kernels). Then, we

https://signetlabdei.github.io/FeSC/ obtained one such figure for each of the 5 outer CV iterations. However, here we report the one providing the best final parameters tuning.

Note that, at each iteration of the outer $\overline{C V}$ loop, we can possibly find a different $\gamma_{r} *$, i.e., $M_{r} *$, and $\mathcal{F}_{r} *$, i.e., specific combination of features (even in case of same $M_{r} *$ value). We can observe that, for a fixed $\nu_{r}$, the accuracy increases with the number $M_{r}$ of features, regardless to the kernel choice. However, a significant improvement is obtained using an rbf kernel. Therefore, in the following, we consider this kernel, only. From Fig. 3. b) we can see that the best parameters tuning in output from the inner $\mathrm{CV}$ (block $C 1$ ) corresponding to one out of 5 outer CV iterations was $\gamma^{*}=[205,0.6,6]$, which achieved an accuracy of 0.7358 . Then, those 205 features were then used for data mining and classification during the corresponding outer $\mathrm{CV}$ iteration. Finally, the combination of features $\mathcal{F}_{r}^{* *}$ and parameters tuning $\gamma_{r}^{* *}$ which provided the best accuracy across all outer $\mathrm{CV}$ iterations were selected as the best model for our classification problem (see Section IV-B). Therefore, the overall training set was used for training a kernel-SVM classifier with the $M_{r}^{* *}$ $\mathcal{F}_{r}^{* *}$ features. Then, the independent (unseen) test set was used to evaluate FeSC performance. Tab. I] summarizes these results. Incidentally, we notice that the best model $\left(\gamma_{r}^{* *}, \mathcal{F}_{r}^{* *}\right)$ does not match the one reported in Fig. 3. In fact, the $\gamma_{r}$ and $M_{r}$ features selected at the output of one inner $\mathrm{CV}$ inner loop might not represent the best model across all outer $\mathrm{CV}$ iterations.

\section{DISCUSSION}

In this work, we propose $\mathrm{FeSC}$ a new feature selection algorithm for IoTH applications, e.g., gesture recognition based on EEG and EMG signals. FeSC is a two-step processing pipeline including: (1) a consensus clustering step to identify the most relevant features to classify two different gestures, e.g., grasping of heavy/light objects, and (2) a data mining step to reduce the dataset for improving classification. We show that our algorithm can classify heavy/light objects using a limited number of features (much smaller than those available in the high-dimensional input feature matrix). We also observe that a proper choice of the SVM s kernel can further improve the classification performance (as reported in Tab. I). Robustness of our results is ensured by the consensus clustering step which selects only those features relevant to all (i.e., two) clustering algorithms and by the nested cross-validation used 
for validation. At the same time, differently from other approaches (e.g., autoencoders [12]), FeSC makes it possible to preserve the physiological meaning of features to allow interpretation by clinical experts. Taking into account only those EEG,EMG sensors leading to relevant features, we could propose a minimal EEG/EMG setup for gesture recognition. This represents a major advantage in this kind of IoTH applications, where individuals are typically more compliant to minimally intrusive sensing platforms. Moreover, limiting the number of sensors for accurate classification of different gestures allows to reduce the amount of data to be stored or transmitted over the IoTH network, with possible savings of, e.g., wireless sensors' batteries, network channel bandwidth, Cloud resources and computational power to instantaneously analyze data. Despite of FeSC]s robustness, our work still suffer from some limitations: the most relevant one is the selection of the specific features to use in the classification. At present, we select the combination of $\{E E G, E M G$, band $\}$ features which shows the best classification accuracy across folds, both in the inner and in the outer CV loops, with the same approach proposed by [15]. However, different strategies could be employed either by selecting a subset of common features across the folds of the inner CV loop [15], or by adding some pre-processing steps to aggregate data in the spatial, i.e., sensors, dimension [11]. Moreover, a larger and more variable dataset could help to gain higher classification performance (preliminary observations over larger datasets, not reported here, shown promising outcomes that need to be confirmed with further investigations). Then, different input features (e.g., partial directed coherence) and additional clustering algorithms for the consensus could be tested to improve classification performance. Finally, the application of FeSC on different datasets, e.g., including different gestures [5], could provide the generalization capabilities of FeSC and can help identifying a common minimal recording setup to use in a variety of out-of-the-lab IoTH applications sharing the same classification target.

\section{CONCLUSIONS}

This work presents FeSC, a new and robust feature selection algorithm to extract the most relevant $\{E E G, E M G$, band $\}$ features for classifying different gestures, i.e., grasping of different objects, while retaining their physiological meaning. FeSC implements a very robust architecture for feature selection and classification. However, our results show that a limited number of features struggles to reach good classification performance, probably due to the limited size of the dataset. In the future, further investigations could determine to what extent size could cause a drop in the performance of FeSC Moreover, we could extend this work to other gestures and, possibly, biosignals in order to extend the applicability of our classification algorithm to new IoTH applications.

\section{ACKNOWLEDGMENTS}

The authors would like to thank Prof. Giorgio Maria Di Nunzio, Dept. Information Engineering, University of Padova, for his support in the pipeline design.

Part of this work was supported by MIUR (Italian Minister for Education) under the initiative "Departments of Excellence" (Law 232/2016).

\section{REFERENCES}

[1] S. R. Islam, D. Kwak, M. H. Kabir, M. Hossain, and K.-S. Kwak, "The internet of things for health care: a comprehensive survey," IEEE Access, vol. 3, pp. 678-708, 2015.

[2] A. Jovic, "Feature selection in biomedical signal classification process and current software implementations," Intelligent Decision Support Systems: Applications in Signal Processing, vol. 4, 2019.

[3] B. Şen, M. Peker, A. Çavuşoğlu, and F. V. Çelebi, "A comparative study on classification of sleep stage based on eeg signals using feature selection and classification algorithms," Journal of medical systems, vol. 38, no. 3, p. 18, 2014.

[4] B. Nakisa, M. N. Rastgoo, D. Tjondronegoro, and V. Chandran, "Evolutionary computation algorithms for feature selection of eegbased emotion recognition using mobile sensors," Expert Systems with Applications, vol. 93, pp. 143-155, 2018.

[5] G. Cisotto, A. V. Guglielmi, L. Badia, and A. Zanella, "Classification of grasping tasks based on EEG-EMG coherence," in 2018 IEEE 20th International Conference on e-Health Networking, Applications and Services (Healthcom), Sep. 2018, pp. 1-6.

[6] S. M. Khan, A. A. Khan, and O. Farooq, "Selection of features and classifiers for emg/eeg based upper limb assistive devices-a review," IEEE reviews in biomedical engineering, 2019.

[7] G. Cisotto, A. V. Guglielmi, L. Badia, and A. Zanella, "Joint Compression of EEG and EMG Signals for Wireless Biometrics," in 2018 IEEE Global Communications Conference (GLOBECOM). IEEE, 2018, pp. 1-6.

[8] D. Ienco and R. Meo, "Exploration and reduction of the feature space by hierarchical clustering," in Proceedings of the 2008 SIAM International Conference on Data Mining. SIAM, 2008, pp. 577587.

[9] H. Liu, X. Wu, and S. Zhang, "Feature selection using hierarchical feature clustering," in Proceedings of the 20th ACM international conference on Information and knowledge management, 2011, pp. 979-984.

[10] J. Cai, J. Luo, S. Wang, and S. Yang, "Feature selection in machine learning: A new perspective," Neurocomputing, vol. 300, pp. 70-79, 2018.

[11] P. Galdi, M. Fratello, F. Trojsi, A. Russo, G. Tedeschi, R. Tagliaferri, and F. Esposito, "Consensus-based feature extraction in rsfMRI data analysis," Soft Computing, vol. 22, no. 11, pp. 37853795, 2018.

[12] A. B. Said, A. Mohamed, T. Elfouly, K. Harras, and Z. J. Wang, "Multimodal deep learning approach for joint EEG-EMG data compression and classification," in 2017 IEEE Wireless Communications and Networking Conference (WCNC). IEEE, 2017, pp. $1-6$.

[13] M. Cerna and A. F. Harvey, "The fundamentals of FFT-based signal analysis and measurement," National Instruments, Junho, vol. 54 2000.

[14] N. V. Chawla, K. W. Bowyer, L. O. Hall, and W. P. Kegelmeyer, "Smote: synthetic minority over-sampling technique," Journal of artificial intelligence research, vol. 16, pp. 321-357, 2002.

[15] S. Parvandeh, H.-W. Yeh, M. P. Paulus, and B. McKinney, "Consensus features nested cross-validation," bioRxiv, pp. 2019-12, 2020.

[16] T. Hastie, R. Tibshirani, and J. Friedman, "Unsupervised learning," in The elements of statistical learning. Springer, 2009, pp. 485585.

[17] W. E. Donath and A. J. Hoffman, "Lower Bounds for the Partitioning of Graphs," IBM J. Res. Dev., vol. 17, no. 5, pp. 420-425, Sep. 1973. [Online]. Available: http: //dx.doi.org/10.1147/rd.175.0420

[18] J. Shi and J. Malik, "Normalized cuts and image segmentation,' IEEE Transactions on Pattern Analysis and Machine Intelligence, vol. 22, no. 8, pp. 888-905, 2000

[19] C. Bishop, "Pattern recognition and machine learning (information science and statistics)," Springer-Verlag New York, Inc. Secaucus, NJ (USA), 2006.

[20] M. D. Luciw, E. Jarocka, and B. B. Edin, "Multi-channel eeg recordings during 3,936 grasp and lift trials with varying weight and friction," Scientific data, vol. 1, p. 140047, 2014. 